\title{
Study of Perfluoroalkyl Chain- Specific Band Shift in Infrared Spectra on the Chain Length
}

\section{AUTHOR(S):}

Shimoaka, Takafumi; Sonoyama, Masashi; Amii, Hideki; Takagi, Toshiyuki; Kanamori, Toshiyuki; Hasegawa, Takeshi

\section{CITATION:}

Shimoaka, Takafumi ...[et al]. Study of Perfluoroalkyl Chain-Specific Band Shift in Infrared Spectra on the Chain Length. The Journal of Physical Chemistry A 2017, 121(44): 84258431

\section{ISSUE DATE:}

2017-11-09

URL:

http://hdl.handle.net/2433/227882

\section{RIGHT:}

This document is the Accepted Manuscript version of a Published Work that appeared in final form in 'The Journal of Physical Chemistry A', copyright (c) American Chemical Society after peer review and technical editing by the publisher. To access the final edited and published work see https://doi.org/10.1021/acs.jpca.7b08940; The full-text file will be made open to the public on 5 October 2018 in accordance with publisher's 'Terms and Conditions for Self-Archiving'; This is not the published version. Please cite only the published version.; この論文は出版社版でありません。引用の際 には出版社版をご確認ご利用ください。 


\section{Study of Perfluoroalkyl Chain-Specific Band Shift in Infrared Spectra on the Chain Length}

Takafumi Shimoaka ${ }^{1}$, Masashi Sonoyama ${ }^{2}$,Hideki Amii ${ }^{2}$, Toshiyuki Takagi ${ }^{3}$, Toshiyuki Kanamori ${ }^{3}$, and Takeshi Hasegawa ${ }^{1 *}$

${ }^{1}$ Laboratory of Chemistry for Functionalized Surfaces, Division of Environmental Chemistry, Institute for Chemical Research, Kyoto University, Gokasho, Uji, Kyoto 611-0011, Japan ${ }^{2}$ Division of Molecular Science, Faculty of Science and Technology, Gunma University, Kiryu, Gunma 376-8515.

${ }^{3}$ National Institute of Advanced Industrial Science and Technology (AIST), AIST Tsukuba Central 5, 1-1-1 Higashi, Tsukuba, Ibaraki 305-8565 
Abstract: 100-200 words

The $\mathrm{CF}_{2}$ symmetric stretching vibration $\left(\mathrm{v}_{\mathrm{s}}\left(\mathrm{CF}_{2}\right)\right)$ band of a perfluoroalkyl (Rf) group in an infrared (IR) spectrum exhibits a unique character, i.e., an apparent high wavenumber shift with increasing the chain length, which is an opposite character to that of the $\mathrm{CH}$ stretching vibration band of a normal alkyl chain. To reveal the mechanism of the unusual IR band shift, two vibrational characters of an Rf chain are focused: (1) a helical conformation of an $\mathrm{Rf}$ chain, (2) the carbon (C) atoms having a smaller mass than the fluorine (F) atom dominantly vibrate as a coupled oscillator leaving $\mathrm{F}$ atoms stay relatively unmoved. These indicate that a 'coupled oscillation of the skeletal $\mathrm{C}$ atoms' of an $\mathrm{Rf}$ chain should be investigated considering the helical structure. In the present study, therefore, the coupled oscillation of the Rf chain dependent on the chain length is investigated by Raman spectroscopy, which is suitable for investigating a skeletal vibration. The Raman active $v_{\mathrm{s}}\left(\mathrm{CF}_{2}\right)$ band is found to be split into two bands, and the splitting is readily explained by considering the helical structure and length with respect to group theory, and the unusual peak shift is concluded to be explained by the helical length. (197 words) 


\section{Introduction:}

The intermolecular interaction between perfluoroalkyl (Rf) chains has recently been revealed to be induced by the dipole-dipole interaction, which is generated by the large dipole moment along each C-F bond. ${ }^{1,2}$ The dipole-dipole interaction induces a close and tight molecular packing of Rf chains in a two dimensional manner, which readily explains the Rf compounds-specific bulk properties represented by the water- and oil-repellency., ${ }^{2,3}$ The molecular packing of $\mathrm{Rf}$ chains is discussed by infrared (IR) spectroscopy ${ }^{4}$ using the peak position of the $\mathrm{CF}_{2}$ symmetric stretching vibration $\left(v_{\mathrm{s}}\left(\mathrm{CF}_{2}\right)\right)$ band at ca. $1150 \mathrm{~cm}^{-1} .2,3$ Thanks to the large dipole moment of the C-F bond, the band strongly appears in an IR spectrum even for a monolayer sample, which makes quantitative discussion easier. Although the IR active $v_{s}\left(C F_{2}\right)$ band is practically useful for analyzing the molecular packing of $\mathrm{Rf}$ compounds, ${ }^{3}$ the band has an unique character of an apparent 'higher wavenumber shift' with increasing the chain length, $n$ (number of $\mathrm{CF}_{2}$ units), i.e., $1126 \mathrm{~cm}^{-1}(n=3), 1135 \mathrm{~cm}^{-1}(n=5), 1146 \mathrm{~cm}^{-1}(n=7), 1149 \mathrm{~cm}^{-1}(n=9)$, and $1151 \mathrm{~cm}^{-1}(n=\infty)$ as presented in Fig. $1,{ }^{2}$ which is an opposite shift to that of the ' $\mathrm{CH}_{2}$ ' symmetric stretching vibration $\left(\mathrm{v}\left(\mathrm{CH}_{2}\right)\right)$ mode. ${ }^{4,5}$ This Rf chain-specific character of the peak shift is, in fact, useful for distinguishing the Rf chain length., ${ }^{2,3}$ However, the mechanism of the unusual shift is not revealed. The aim of the present study is thus revealing the origin of the unusual Rf-specific shift. 


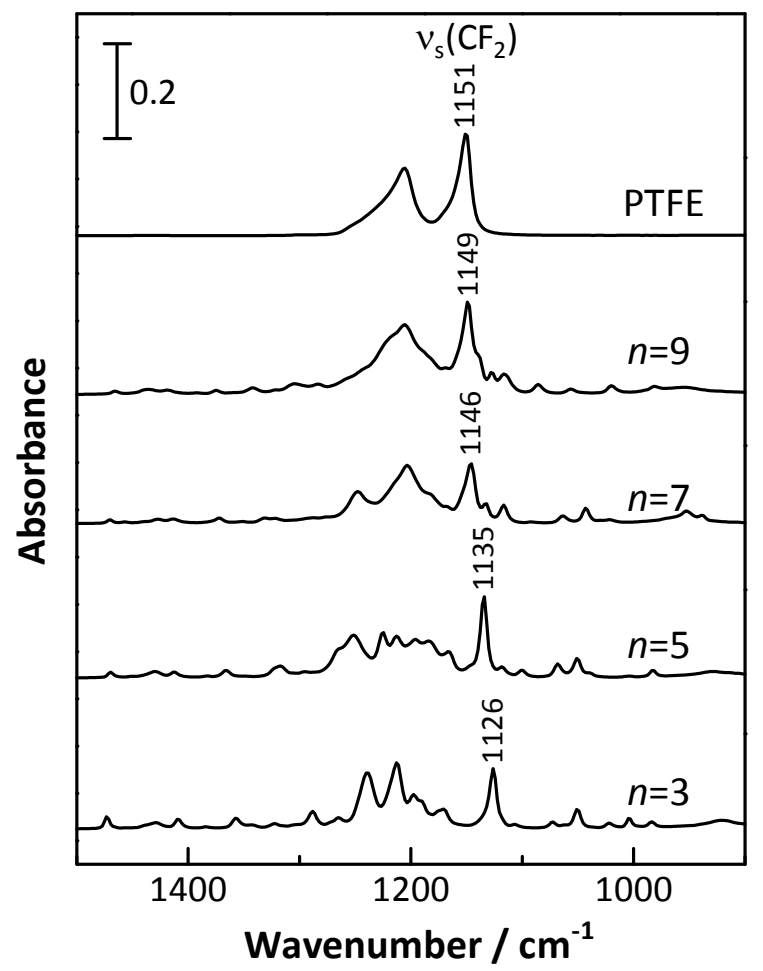

Fig. 1 The C-F stretching vibration region of IR spectra of an $\mathrm{Rf}$ chain with different lengths measured by using the ATR technique with an ATR prism of Ge at the angle of incidence of $45^{\circ} .^{2}$ The $v_{\mathrm{s}}\left(\mathrm{CF}_{2}\right)$ band exhibits a unique high-wavenumber shift with the Rf length.

On considering the vibration of a molecular chain composed of many repeat units, the coupled oscillation has to be considered. ${ }^{6}$ Although the shift of the $v_{\mathrm{s}}\left(\mathrm{CF}_{2}\right)$ band should depend on the shape of the coupled oscillation as commonly found for a normal alkyl chain, the vibration of an $\mathrm{Rf}$ chain has a complicated character as follows. The coupled oscillation of the $\mathrm{CH}_{2}$ symmetric stretching vibration $\left(v_{\mathrm{s}}\left(\mathrm{CH}_{2}\right)\right)$ mode of a normal alkyl chain is explained briefly, followed by discussion of the difference of the $v_{\mathrm{s}}\left(\mathrm{CF}_{2}\right)$ mode from $v_{\mathrm{s}}\left(\mathrm{CH}_{2}\right)$, so that the mechanism of the shift would be revealed. 
Coupled Oscillation of a Normal Alkyl Chain. Molecular structure of a normal alkyl chain having the all-trans zigzag conformation corresponds to the point group of $D_{2 \mathrm{~h}}$, on which the vibration is analyzed by the factor group analysis. ${ }^{5,7-13}$ On the discussion of the coupled oscillation of the alkyl chain, the phase difference $(\phi)$ between adjacent two $\mathrm{CH}_{2}$ units is crucial. ${ }^{6}$ The coupled oscillation has many shapes on the phase $(0 \leq \phi \leq \pi)$, and the frequency of the $v_{\mathrm{s}}\left(\mathrm{CH}_{2}\right)$ mode dependent on $\phi$ (dispersion curve) is revealed by the GF calculation method. ${ }^{11}$ Fortunately, Raman and IR spectroscopies only observe the vibration bands of $\phi=0$ and $\phi=\pi$, respectively, both of which corresponds to the wavenumber vector of nil $(k=0)$ because of the restriction by the Brillouin-center condition. The schematics of the $v_{\mathrm{s}}\left(\mathrm{CH}_{2}\right)$ modes of an ethylene unit having $\phi=0$ and $\phi=\pi$ are presented in Fig. 2a and b. When a methylene group alone in the schematics is focused, two hydrogen $(\mathrm{H})$ atoms seem oscillate

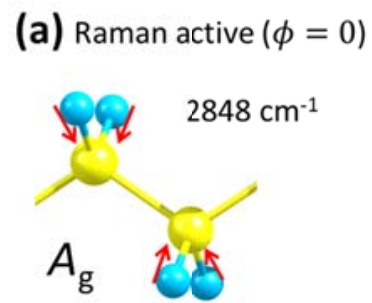

(b) IR active $(\phi=\pi)$

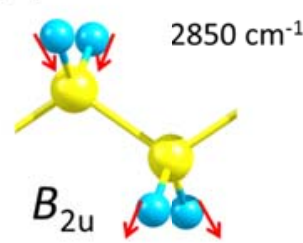

(c) Raman active $(\phi=0)$

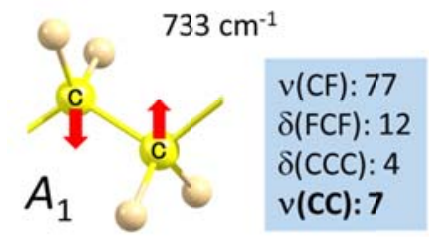

(d) IR active $(\phi=\pi)$

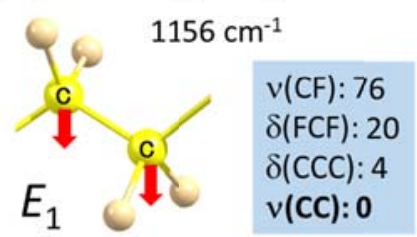

Fig. 2 Schematic images of the coupled oscillation of an alkyl chain having the phase difference of (a) $\phi=0$ and (b) $\phi=\pi$. Those of an Rf chain having (c) $\phi=0$ and (d) $\phi$ $=\pi$, and the potential energy distribution (\%) of vibrational components in the vibration modes (c) and (d) reported by Hannon et al (Ref. \#22). 
with the same phase, which is thus denoted as the $v_{\mathrm{s}}\left(\mathrm{CH}_{2}\right)$ mode. As a matter of fact, this vibration mode is of a repeat unit of $\mathrm{CH}_{2}$, however, which has different shapes on the wavenumber vector or the phase, and they are assigned to different symmetry species: the former vibration of $\phi=0$ is assigned to the Raman-active $A_{\mathrm{g}}$ mode, and the other $(\phi=\pi)$ is assigned to the IR-active $B_{2 \mathrm{u}}$ mode.

The vibrational difference on $\phi$ influences the peak position. In fact, the $v_{\mathrm{s}}\left(\mathrm{CH}_{2}\right)$ bands of polyethylene in Raman and IR spectra appear at slightly different positions of 2848 and $2850 \mathrm{~cm}^{-1}$, respectively. ${ }^{4,10}$ This non-coincidence is practically found in the spectrum of a normal alkane, and the peak exhibits a lower wavenumber shift with increasing the chain length ${ }^{5}$ because of the decrease of the elements in the matrix of $\mathrm{G}$ of the GF-matrix method. ${ }^{14,15}$

It sounds strange that the observed difference of the wavenumber positions of $\phi=0$ and $\pi$ is only ca. $2 \mathrm{~cm}^{-1}$, although the shapes of the two oscillations are largely different from each other. This is because the mass of hydrogen $(\mathrm{H})$ is much less than that of carbon $(\mathrm{C})$, i.e., the oscillation is intrinsically represented by $\mathrm{H}$ atoms, and $\mathrm{C}$ atoms are relatively unmoved. On considering the $v_{\mathrm{s}}\left(\mathrm{CF}_{2}\right)$ mode, the situation should change greatly, since the mass of fluorine $(\mathrm{F})$ is larger than that of $\mathrm{C}$. In fact, the $v_{\mathrm{s}}\left(\mathrm{CF}_{2}\right)$ mode appears at ca. 1150 and $730 \mathrm{~cm}^{-1}$ in IR and Raman spectra, respectively, and the difference is fairly large by more than $400 \mathrm{~cm}^{-1}$. This implies that the coupled-oscillation phenomena of an $\mathrm{Rf}$ group should be significantly different from those of a normal alkyl group.

Coupled Oscillation of an Rf chain. The critical difference of $\mathrm{F}$ from $\mathrm{H}$ is not only for elemental mass, but also for the radii of the atoms. ${ }^{2,3,16}$ The larger radii of $\mathrm{F}$ than $\mathrm{H}$ by ca. $20 \%$ induces the $\mathrm{Rf}$ 
chain-specific helical structure due to the steric repulsion between the adjacent $\mathrm{CF}_{2}$ units. The twisting rate of the Rf chain depends on the phase diagram of polytetrafluoroethylene (PTFE known as Teflon $\left.^{\circledR}\right) \cdot{ }^{17-20}$ At an ambient temperature in the phase IV $\left(19 \sim 30^{\circ} \mathrm{C}\right)$, the Rf chain has a symmetry of the $15_{7}$-helix about the chain axis, i.e., the direction of the symmetric axis of the $\mathrm{CF}_{2}$ unit is rotated by $180^{\circ}$ over $15 \mathrm{CF}_{2}$ units. This helical structure corresponds to the point group of $D_{15}$.

The vibrational modes of PTFE have also been studied already by the factor group analysis using $D_{15}{ }^{21-24}$ The point group of $D_{15}$ has nine irreducible representations, $A_{1}, A_{2}, E_{1}, E_{2}, \cdots, E_{7}$, and the irreducible representations of IR and Raman spectroscopies are calculated to be: $\Gamma_{\text {vib }}^{\mathrm{IR}}=3 A_{2}+8 E_{1}$ and $\Gamma_{\text {vib }}^{\text {Raman }}=4 A_{1}+8 E_{1}+9 E_{2}$, respectively. The frequencies of the vibrational modes of PTFE were evaluated theoretically by the GF calculation using a classical force constant, ${ }^{22-24}$ and the $v_{\mathrm{s}}\left(\mathrm{CF}_{2}\right)$ band at ca. $1150 \mathrm{~cm}^{-1}$ in an IR spectrum of PTFE is assigned to the IR-active mode of $\phi=\pi\left(E_{1}\right)$ as presented in Fig. 2d. The dispersion curve of the $v_{\mathrm{s}}\left(\mathrm{CF}_{2}\right)$ mode is also reported, and the Raman band at ca. 730 $\mathrm{cm}^{-1}$ is assigned to the Raman-active $v_{\mathrm{s}}\left(\mathrm{CF}_{2}\right)$ band $(\phi=0) .{ }^{22-24}$ The schematic of the Raman mode is presented in Fig. 2c.

The difference of relative mass of $\mathrm{F}$ and $\mathrm{H}$ to carbon (C) changes the shape of the normal mode greatly. Contrary to the $\mathrm{CH}$ stretching vibration modes, the $\mathrm{C}$ atoms having a smaller mass than the $\mathrm{F}$ dominantly vibrate leaving the F atoms stay relatively unmoved as presented in Fig. $2 \mathrm{c}$ and $\mathrm{d}$. Since the $\mathrm{C}$ atoms in the $\mathrm{Rf}$ chain are covalently bonded with the single bonds $(-\mathrm{C}-\mathrm{C}-)$, the 'coupled oscillation of the skeletal $C$ atoms' must be considered. The character specific to the coupled oscillation of the 
skeleton has already been pointed out for the $\mathrm{CF}_{3}$ symmetric stretching vibration $\left(v_{\mathrm{s}}\left(\mathrm{CF}_{3}\right)\right)$ mode. ${ }^{25}$ Although the $\mathrm{CH}_{3}$ symmetric stretching vibration $\left(v_{\mathrm{s}}\left(\mathrm{CH}_{3}\right)\right)$ mode of a normal alkyl chain is nearly localized at the terminate methyl group, the $v_{\mathrm{s}}\left(\mathrm{CF}_{3}\right)$ mode is spread over the entire $\mathrm{Rf}$ group because of the coupled oscillation, which means that the transition moment of the $v_{\mathrm{s}}\left(\mathrm{CF}_{3}\right)$ mode is parallel to the $\mathrm{Rf}$ chain. ${ }^{25}$ As a result, the intensity of the $v_{s}\left(C F_{3}\right)$ band is useful for discussing the orientation of Rf chains in an thin film..$^{2,3,25}$ In this manner, investigation of the coupled oscillation of the skeletal $\mathrm{C}$ atoms is crucial to reveal the unusual IR-active $v_{\mathrm{s}}\left(\mathrm{CF}_{2}\right)$ band shift.

Aim of the Present Study. To reveal the origin of the unusual IR shift dependent on the Rf length, the $v_{\mathrm{s}}\left(\mathrm{CF}_{2}\right)$ band is investigated by using 'Raman' spectroscopy, which is suitable for investigating a skeletal vibration. As the samples, myristic acid derivatives having an $\mathrm{Rf}$ group $\left(\mathrm{CF}_{3}\left(\mathrm{CF}_{2}\right)_{n}\left(\mathrm{CH}_{2}\right)_{m}-\mathrm{COOH}\right.$; MA-Rfn where $n=3,5,7,9$ and $\left.n+m=12\right)$ were chosen as used in a previous work. $^{3,25}$ The IR spectra shown in Fig. $1(n=3 \sim 9)$ are the results of these compounds. As a result, the Raman active $v_{\mathrm{s}}\left(\mathrm{CF}_{2}\right)$ band is split into two bands for $n=7$ or shorter. By considering the Rf chain structure in terms of group theory, the splitting is readily explained by the conformational change of the Rf group. After a spectral simulation based on density functional theory (DFT), the lower-wavenumber peak is assigned to the Raman active $v_{\mathrm{s}}\left(\mathrm{CF}_{2}\right)$ band, which exhibits intrinsically the same shift as the IR-active $v_{\mathrm{s}}\left(\mathrm{CF}_{2}\right)$ band. The unusual peak shift is concluded to be explained by this conformational change. 


\section{Experimental:}

11,11,12,12,13,13,14,14,14-Nonafluorotetradecanoic

9,9,10,10,11,11,12,12,13,13,14,14,14-tridecafluorotetradecanoic acid

(MA-Rf3),

acid

(MA-Rf5),

7,7,8,8,9,9,10,10,11,11,12,12,13,13,14,14,14-heptadecafluorotetradecanoic acid (MA-Rf7) and 5,5,6,6,7,7,8,8,9,9,10,10,11,11,12,12,13,13,14,14,14-henicosafluorotetra-decanoic acid (MA-Rf9) were synthesized as reported in our previous work. ${ }^{3}$ A PTFE tape was purchased from AS ONE Corporation (Tokyo, Japan).

Raman spectra were measured by using a Thermo Fischer Scientific (Madison, WI, USA) DXRxi Raman imaging microscope having a diode-pumped laser with an excitation wavelength of $532 \mathrm{~nm}$, a grating having 900 lines $\mathrm{mm}^{-1}$ and an edge filter for eliminating the Rayleigh scattered light. On this spectrometer, Raman spectra in the range of $3500-50 \mathrm{~cm}^{-1}$ with the resolution of $5 \mathrm{~cm}^{-1}$ are measured. The bulk sample of MA-Rfn in the solid state was put on a glass plate, and an objective lens of 10x with a confocal aperture with a pinhole of $25 \mu \mathrm{m}$ width were used for both laser irradiation and collection of the Raman scattered light. The laser spot size is $10 \mu \mathrm{m}$, which corresponds to the spatial resolution (xy) limit on the condition, and it also determines the depth resolution (z), $30 \mu \mathrm{m}$. Since the linearly polarized light was irradiated on the sample, the band intensity should be influenced by molecular orientation. In the present study, however, it has no problem because only the band position is discussed. The laser was operated at a constant power of $10.0 \mathrm{~mW}$ at the sample surface. The Raman spectrum was obtained with an exposure time of $4 \mathrm{~s}$, and with a total acquisition time of 150 seconds. 
Density functional calculation for a spectral simulation was carried out by using the Gaussian 09 program. ${ }^{25}$ The optimal geometry, harmonic wavenumbers and Raman intensities of a single MA-Rfn molecule were obtained using 6-311++G basis set. For the DFT calculations, Becke's three-parameter exchange functional together with correlation functional of Lee-Yang-Parr (B3LYP) was employed. ${ }^{27,28}$ A harmonic wavenumber, $\tilde{v}_{\text {harm }}$, was corrected by the linear scaling factor, $\tilde{v}_{\text {scaled : }}$ $\tilde{v}_{\text {scaled }}=0.9613 \cdot \tilde{v}_{\text {harm }} \cdot{ }^{29}$ 


\section{Results and Discussion}

\section{Splitting of the Raman-active $v_{s}\left(C_{2}\right)$ Bands of Partially Fluorinated Myristic Acids:}

Fig. 3 presents Raman spectra of MA-Rfn $\left(n=3,5,7\right.$ and 9) at an ambient temperature $\left(>19{ }^{\circ} \mathrm{C}\right)$, which corresponds to the phase IV. As a sample of an extremely long Rf chain $(n=\infty)$, a PTFE tape was also measured. As mentioned in Introduction, the helical structure of the $\mathrm{Rf}$ chain in the phase IV has the symmetry represented by the point group of $D_{15}$. The Raman peaks observed in the spectrum of PTFE are, thus, assigned to the Raman-active vibration modes of $D_{15}\left(A_{1}, E_{1}\right.$, and $\left.E_{2}\right)$ as shown in Fig. 3.

Since MA-Rfn is a diblock compound consisted of the Rf and normal alkyl parts, Raman spectra of MA-Rfn also have bands derived from the normal alkyl part, which are highlighted by blue; whereas the bands derived from the Rf part are highlighted by pink. The lower two peaks (ca. $384 \mathrm{~cm}^{-1}$ and ca. 290

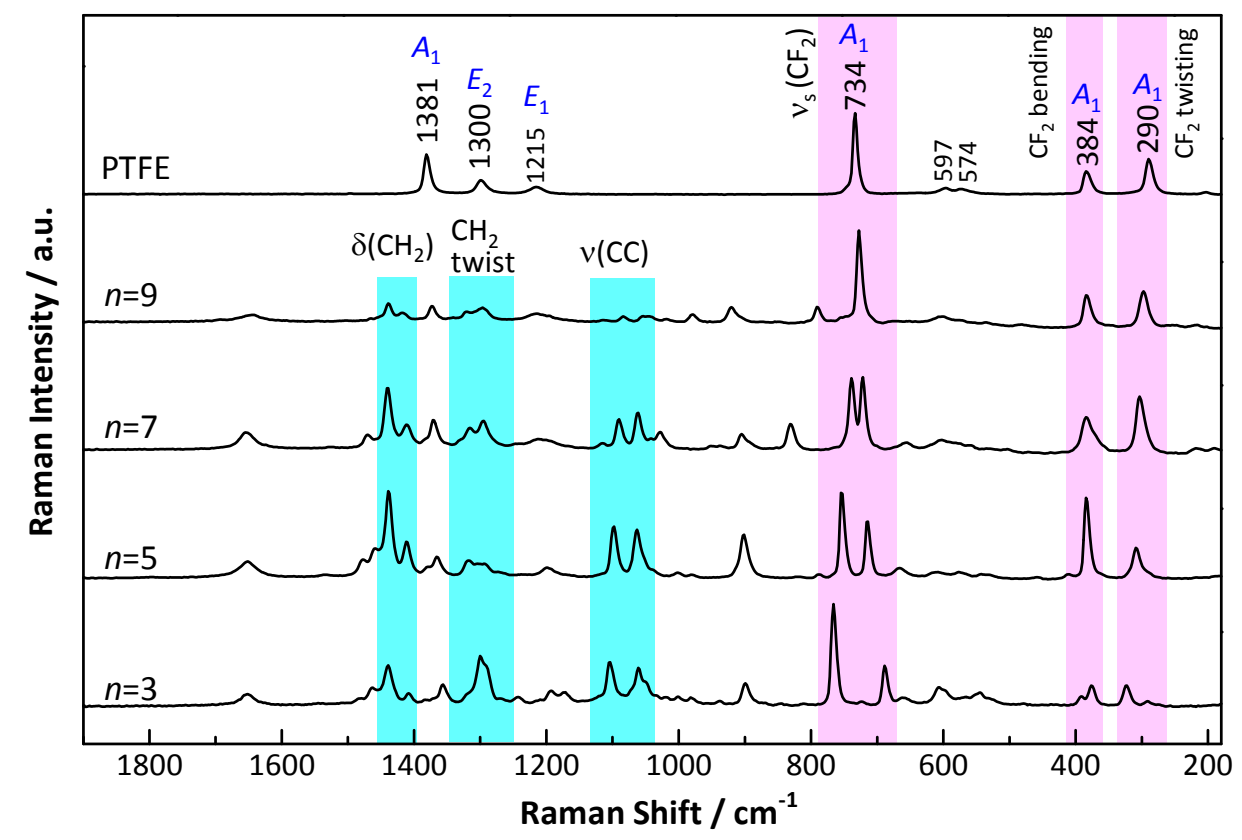

Fig. 3 Raman spectra of MA-Rfns $(n=3,5,7$ and 9) and a PTFE tape. 
$\mathrm{cm}^{-1}$ ) are assigned to the $\mathrm{CF}_{2}$ bending and twisting vibration bands, respectively. ${ }^{22}$ The band at ca. 730 $\mathrm{cm}^{-1}$ is the key band for the present study, which is assigned to the Raman-active $v_{\mathrm{s}}\left(\mathrm{CF}_{2}\right)$ mode with $\phi=$ $0\left(A_{1}\right)$. This band exhibits a remarkable change that the peak is split into two peaks for $n=7$, and the splitting width becomes larger with decreasing $n$. The mechanism of the band splitting is discussed as follows.

The splitting is known to appear for a coupled oscillator composed of repeat units having a high symmetry as found in a normal alkyl chain with $D_{2 \mathrm{~h}}$, which includes the mirror (or glide) plane as a symmetry element, because all the $\mathrm{C}$ atoms are in a plane. The factor group analysis on $D_{2 \mathrm{~h}}$ reveals that a normal alkyl chain has two skeletal vibration modes, in which the $\mathrm{C}-\mathrm{C}$ bond length changes without a significant change of the $\mathrm{C}-\mathrm{H}$ bond length. ${ }^{7}$ One of the two modes has the symmetry species of $A_{\mathrm{g}}$, in which the $\mathrm{C}$ atoms vibrates in the radial direction ( $y$ direction) with $\phi=0$ as presented in Fig. $4 \mathrm{a}$. The other mode is the $B_{1 \mathrm{~g}}$ mode, in which the $\mathrm{C}$ atoms vibrate nearly along the chain in the $x-y$ plane (Fig. 4b). Both modes are denoted as the $\mathrm{C}-\mathrm{C}$ stretching vibration $\mathrm{v}(\mathrm{CC})$ mode. In fact, MA-Rfn having a relatively short Rf chain $(n=3,5,7)$ has a long 'normal alkyl' part, which yields the two $v(C C)$ bands at ca. $1100\left(A_{\mathrm{g}}\right)$ and $1060 \mathrm{~cm}^{-1}\left(B_{1 \mathrm{~g}}\right)\left(\right.$ Fig. 3). In this manner, the $D_{2 \mathrm{~h}}$ symmetry is the origin of the band splitting of a $v(\mathrm{CC})$-related vibration band. Since the mass of $\mathrm{C}$ is smaller than $\mathrm{F}$, as mentioned above, the carbon chain works as the core part of a normal mode on an $\mathrm{Rf}$ chain, which is true of the $v_{\mathrm{s}}\left(\mathrm{CF}_{2}\right)$ mode as mentioned below. 

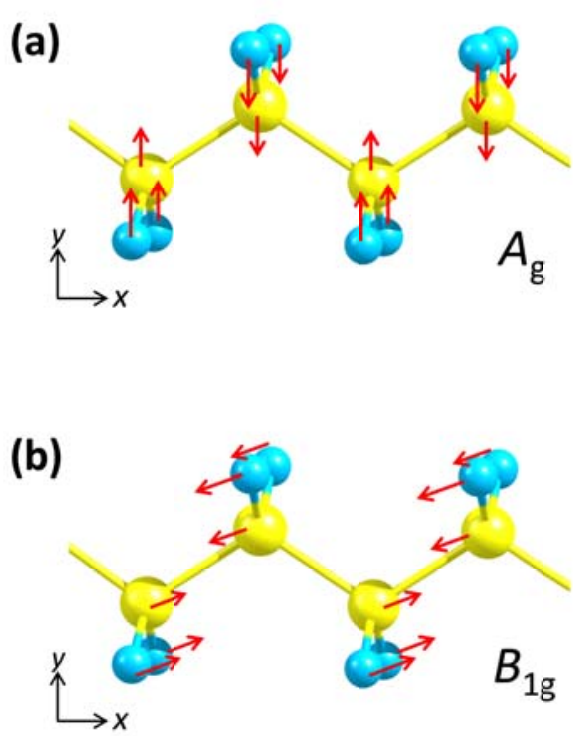

Fig. 4 Schematic image of the skeletal vibration $(v(C C))$ of an alkyl chain: (a) $A_{g}$ and (b) $B_{1 \mathrm{~g}}$ modes.

Fortunately, a former study using the GF calculation of PTFE reveals the potential energy distribution (\%) of vibrational components in both IR and Raman-active $v_{\mathrm{s}}\left(\mathrm{CF}_{2}\right)$ modes, ${ }^{22}$ which are presented in the squares in Fig. $2 \mathrm{c}$ and d. The IR-active $E_{1}$ mode (Fig. 2d) accompanies the F-C-F and $\mathrm{C}-\mathrm{C}-\mathrm{C}$ bending vibrations $\left(\delta(\mathrm{FCF})\right.$ and $\delta(\mathrm{CCC})$, respectively). The Raman-active $A_{1}$ mode (Fig. 2c) accompanies an additional vibration component of the $\mathrm{C}-\mathrm{C}$ stretching vibration $(\mathrm{v}(\mathrm{CC}))$ as well as the $\delta(\mathrm{FCF})$ and $\delta(\mathrm{CCC})$ vibrations. These details of the $v_{\mathrm{s}}\left(\mathrm{CF}_{2}\right)$ mode are comprehensively understood by considering the $v(\mathrm{CC})$ vibration as a key vibration. The $v_{\mathrm{s}}\left(\mathrm{CF}_{2}\right)$ vibration is thus a result induced by the $v(\mathrm{CC})$ vibration, and the $\delta(\mathrm{FCF})$ and $\delta(\mathrm{CCC})$ vibrations are also generated as vice products. In other words, the essence of the $v_{\mathrm{s}}\left(\mathrm{CF}_{2}\right)$ mode is of the $v(\mathrm{CC})$ vibration over the Rf group, and the name of 'the $v_{\mathrm{s}}\left(\mathrm{CF}_{2}\right)$ mode' is a 'representation of a local image' of the $v_{\mathrm{s}}\left(\mathrm{CF}_{2}\right)$ vibration at a $\mathrm{CF}_{2}$ group. This 
vibrational image agrees with the unusual motion of an $\mathrm{Rf}$ group considering the larger mass of $\mathrm{F}$ than that of $\mathrm{C}^{25}$

Here, note that a short $\mathrm{Rf}$ group having three $\mathrm{CF}_{2}$ groups is known to have a planer skeleton with $D_{2 \mathrm{~h}}{ }^{16}$ If the vibration of an $\mathrm{Rf}$ group is driven by the skeleton vibration, therefore, MA-Rf3 should exhibit doubly-split bands for the $v_{\mathrm{s}}\left(\mathrm{CF}_{2}\right)$ mode, which is confirmed in Fig. 5a. On the other hand, if the symmetry of the molecular skeleton is lowered, the splitting is lost to have a single band, which readily explains the single $v_{\mathrm{s}}\left(\mathrm{CF}_{2}\right)$ band at $734 \mathrm{~cm}^{-1}$ for PTFE having the symmetry of $D_{15}$.

It is worth noting that the other compounds of MA-Rfn having intermediate Rf lengths $(n=5 \sim 9)$ exhibit a gradual change of the splitting. To discuss the splitting width depending on the Rf length $(n)$,

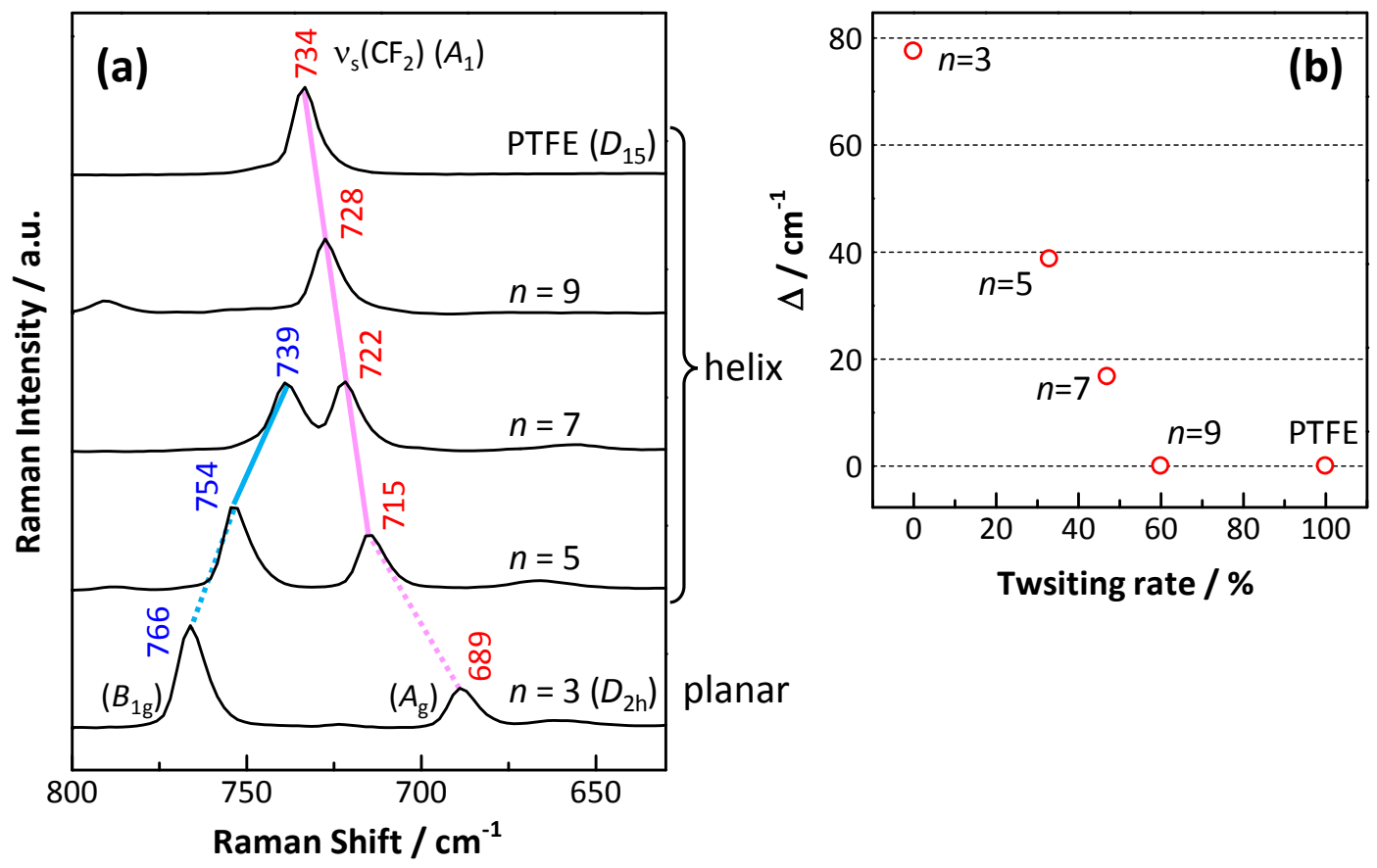

Fig. 5 (a) Magnified spectra in the Raman-active $v_{\mathrm{s}}\left(\mathrm{CF}_{2}\right)$ region. (b) Splitting width between the two bands shown in the $v_{\mathrm{s}}\left(\mathrm{CF}_{2}\right)$ region $(\Delta)$ dependent on $n$. 
the 'twisting rate' of the Rf chains is introduced for convenience. Twisting angles of the two $\mathrm{CF}_{2}$ groups at both ends of the $\mathrm{Rf}$ chain with $n=9,7$ and 5 are $108^{\circ}, 84^{\circ}$ and $60^{\circ}$, respectively, and the 'twisting rate' $(\%)$ is calculated, so that the angle of $n=15\left(180^{\circ}\right)$ corresponds to $100 \%$ as presented in the third column of Table 1. In addition, since the Rf chain with $n=3$ has exceptionally the all-trans zigzag conformation, the twisting rate for $n=3$ is made $0 \%$. The splitting width $(\Delta)$ against the twisting rate is plotted as presented in Fig. 5b.

Table 1 Twisting rate of $\mathrm{Rf}$ chain dependent on the chain length

\begin{tabular}{ccc} 
Number of & Twisted angle of the two & Twisting rate $/ \%$ \\
$\mathrm{CF}_{2}$ units: $n$ & $\mathrm{CF}_{2}$ group at both ends $/{ }^{\circ}$ & \\
\hline 3 & 0 & 0 \\
5 & 60 & 33 \\
7 & 84 & 47 \\
9 & 108 & 60 \\
$\vdots$ & $\vdots$ & $\vdots$ \\
15 & 180 & 100 \\
\hline
\end{tabular}

The spectrum of PTFE and $n=9$ has a single $v_{\mathrm{s}}\left(\mathrm{CF}_{2}\right)$ band, and thus $\Delta$ is zero. It is interesting that $\Delta$ in the $n=3 \sim 9$ is found to have a 'linear correlation' with the twisting rate. This indicates that the splitting width reflects the conformational change of the skeleton from the planer structure $\left(D_{2 \mathrm{~h}}\right)$ to the helical structure $\left(D_{15}\right)$. Through this analysis, the Rf chain having $n=9$ is found to have a vibrational character, as if the helix were completed, although the twisting rate is only $60 \%$. This implies that the $v_{\mathrm{s}}\left(\mathrm{CF}_{2}\right)$ mode of MA-Rf5 and 7 has an intermediate character between the planer structure $\left(D_{2 \mathrm{~h}}\right)$ and the 
helical structure $\left(D_{15}\right)$ as the molecular vibration. In the next section, the assignment of the two bands is mentioned, and the origin of the unusual peak shift in IR spectra is revealed.

Assignment of the two bands in the $v_{s}\left(C_{2}\right)$ region and the origin of the peak shift of the IR-active $v_{s}\left(\mathbf{C F}_{2}\right)$ band

To make the assignment of the two Raman bands in the $v_{\mathrm{s}}\left(\mathrm{CF}_{2}\right)$ region, QC calculations were carried out. The calculated Raman spectra of MA-Rfn $(n=3,5,7$ and 9) are presented in Fig. S1 in Supporting Information, which are compared with the measured spectra. Almost all the measured peaks are readily reproduced by the calculation. Of course, the calculated spectrum for $n=9$ has a single band in the $v_{\mathrm{s}}\left(\mathrm{CF}_{2}\right)$ region, and the vibrational motion is thus assigned to the $A_{1}$ mode such as the schematic image shown in Fig. 2c, i.e., the chain of $\mathrm{C}$ atoms vibrates in the perpendicular (radial) direction to the $\mathrm{Rf}$ chain axis. The calculated spectra shown in Fig. S1 also reproduce the band splitting for $n=7$ or shorter, and MA-Rf3 exhibits the largest splitting. The vibrational motion of the lower wavenumber band presented in Fig. 5a indicates that the center $\mathrm{C}$ atoms (2nd and 3rd) in the Rf chain vibrate nearly along the $y$ direction with $\phi=0$ (Fig. 6a). Therefore, the lower band at $689 \mathrm{~cm}^{-1}$ is assignable to the symmetry species of $A_{\mathrm{g}}$ of $D_{2 \mathrm{~h}}$. On the other hand, the other band at $766 \mathrm{~cm}^{-1}$ corresponds to the vibrational motion presented in Fig. 6b, i.e., the $\mathrm{C}$ atoms at the both ends (1st and 4th) of the Rf chain vibrate in the $x-y$ plane, which is assignable to $B_{1 \mathrm{~g}}$. As a result, the two $v_{\mathrm{s}}\left(\mathrm{CF}_{2}\right)$ bands for $n=3$ have the same symmetry species $\left(A_{\mathrm{g}}\right.$ and $\left.B_{1 \mathrm{~g}}\right)$ as those of the skeletal vibration of a normal alkyl chain presented in Fig. 4 . Since 
(a)

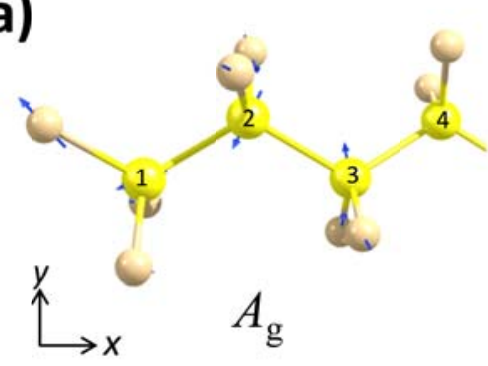

(b)

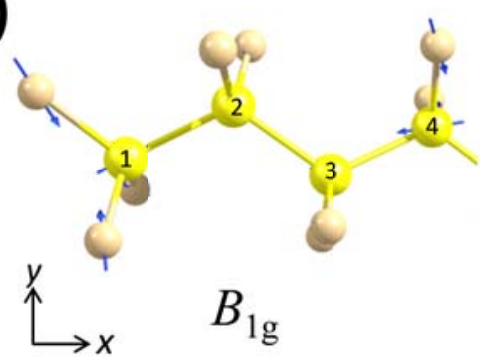

Fig. 6 The calculated vibrational motions of the split two bands for $n=3$ : (a) 689 and (b) $766 \mathrm{~cm}^{-1}$.

the $B_{1 \mathrm{~g}}$ mode is Raman-active but IR-'inactive', the split two peaks are available only in Raman spectra; whereas the IR spectra have only the lower wavenumber band (Fig. 2d).

Although the entire Rf chain of $n=5$ and 7 has the helical conformation, the two bands in the $v_{\mathrm{s}}\left(\mathrm{CF}_{2}\right)$ region for $n=5$ and 7 suggest that the vibrational mode involves the planer character due to the low twisting rate. To confirm this speculation, the calculated result of $n=7$ is discussed as an example. The vibrational mode of the lower wavenumber band at $722 \mathrm{~cm}^{-1}$ (Fig. 5a) is assigned to the $A_{1}$ mode as presented in Fig. S2a. The image from top view (Fig. S2a') clearly indicates that the C atoms vibrate in the radial direction of the chain. In the same manner as $n=3$, the $\mathrm{C}$ atoms in the center (marked by the pink square) mainly vibrate; whereas those at both ends have very minor motion. In the vibrational motion of the higher wavenumber band at $739 \mathrm{~cm}^{-1}$ presented in Fig. S2b, on the other hand, the C atoms on both ends (marked by the blue square) vibrate with a large amplitude 'along' the Rf chain. On closer inspection, the arrows on the three $\mathrm{C}$ atoms at each end lie in the plane involving the three $\mathrm{C}$ atoms. This indicates that the individually vibrating part (marked by the blue square) belongs to $D_{2 \mathrm{~h}}$. 
The individually vibrating part has a motion assigned to the Raman-'inactive' $B_{3 u}$ mode. When the entire molecule is taken into account, however, $\mathrm{C}$ atoms in the two squares vibrate in the opposite direction. Therefore, the vibration mode of the entire Rf chain is assigned to the Raman-active totally symmetric $A_{1}$ mode of $D_{15}$ along the chain direction. As a result, the higher wavenumber band is thus concluded to be induced by the planer structure. The two $v_{\mathrm{s}}\left(\mathrm{CF}_{2}\right)$ bands for $n=5$ are readily explained in the same fashion.

The $A_{1}$ mode is 'IR-inactive,' which readily explains the single IR band in the $v_{\mathrm{s}}\left(\mathrm{CF}_{2}\right)$ region for $n=$ 7 as well as $n=3$. As a result, the lower wavenumber bands for $n=3 \sim 7$ are all explained by the $A_{1}$ mode in terms of the vibration of the carbon atoms in the radial-direction. The higher wavenumber band shift (indicated by the pink line in Fig. 5a) with increasing $n$ corresponds to the increasing $\Delta$ of the split 'two' bands in Raman spectra.

The higher wavenumber shifts of the Raman $\left(A_{\mathrm{g}}\right)$ and IR $\left(E_{1}\right) v_{\mathrm{s}}\left(\mathrm{CF}_{2}\right)$ bands are interesting, if the band positions are compared as plotted in Fig. 7 against the twisting rate. The Raman band positions are presented by the green circles (see the right axis). The change is gradual along the green dashed curve, which shows a good correlation between the Raman band and the twisting rate. This indicates that the correspondence of $n=\infty$ to $100 \%$ is reasonable, and the Rf chain of $n=3$ certainly has the planer structure. Since the helical structure generates a strain in the carbon skeleton, the higher peak shift can also be interpreted as an increased strain of the $\mathrm{Rf}$ chain as a result of an increased force constant of the Rf chain. 


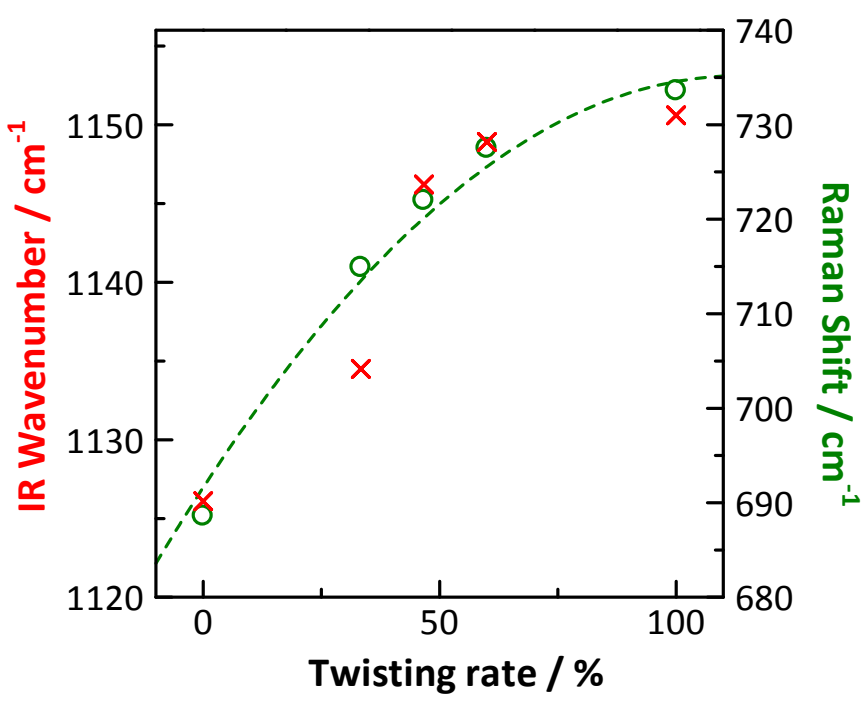

Fig. 7 The IR-(red, left axis) and Raman-active $v_{\mathrm{s}}\left(\mathrm{CF}_{2}\right)$ band positions (green, right axis) plotted against the twisting rate.

On the other hand, the $v_{s}\left(\mathrm{CF}_{2}\right)$ band positions of IR spectra shown in Fig. 1 are plotted by the red cross (see the left axis) in Fig. 7 exhibits a similar trend to the Raman band except an apparent wavenumber jump between $33 \%(n=5)$ and $47 \%(n=7)$. The entire trend is similar to that of Raman, which can be explained by the representative vibrational components commonly involved in the Ramanand IR-active modes as summarized beside Fig. $2 \mathrm{c}$ and d. On the other hand, the IR-specific apparent jump is attributed to a fundamental difference between Raman and IR spectroscopies.

The Raman bands are derived from the molecular polarizability change of the $\mathrm{Rf}$ chain; whereas the IR bands depend on a change of the transition dipole moment. Therefore, the IR band strongly reflects an atmosphere of a dipole. In our previous study, the aggregation property of MA-Rfn dependent on $n$ was studied by using IR spectroscopy. The study revealed that MA-Rf $n$ having $n=7$ or longer forms a 
close molecular packing via the strong dipole-dipole interaction; whereas those having $n=5$ or shorter exhibit a poor packing. ${ }^{2,3,30}$ Since the strong dipole-dipole interactions make the molecules tightly aggregated only for $n=7$ or longer, the atmosphere about a C-F bond would largely change from $n=5$ to 7. In other words, the difference of the dipole's atmosphere induces a discontinuous IR band shift between $n=5$ and 7. In short, IR bands of an Rf compound are influenced not only the skeletal structure, but also the packing of the $\mathrm{Rf}$ chain, which is a benefit of using IR spectroscopy for investigating the packing. 


\section{Summary:}

To reveal the Rf-compound specific IR band shift of the $v_{s}\left(C F_{2}\right)$ dependent on the $\mathrm{Rf}$ chain length, $n$ (number of $\mathrm{CF}_{2}$ units: $n=3,5,7$ and 9), the skeletal vibration of the $\mathrm{Rf}$ chain has been investigated by using Raman spectroscopy. While the Raman-active $v_{\mathrm{s}}\left(\mathrm{CF}_{2}\right)$ band of $n=9$ has a single band at ca. $730 \mathrm{~cm}^{-1}$ as well as PTFE, the band is split into two bands for $n=3 \sim 7$, and the splitting becomes larger with decreasing $n$. To investigate the origin of the splitting, a 'twisting rate' of the Rf chain is introduced, which is a useful index for interpreting the conformational change with $n$. The band splitting width was found to have a good correlation with the twisting rate, and the variation of the width was readily attributed to the conformational change between $D_{15}$ and $D_{2 \mathrm{~h}}$. The 'lower' wavenumber band of the split two bands in the spectrum of $n=3 \sim 7$ corresponds to the $v_{\mathrm{s}}\left(\mathrm{CF}_{2}\right)$ band, which shows a similar higher wavenumber shift to the IR band. The $v_{s}\left(C F_{2}\right)$ band position also has a good correlation with the twisting rate, and the mechanism of the unusual shift has been concluded to be attributed to the 'strain' of the skeletal chain dependent on the chain length. A similar trend is found for the IR band position. Since the 'higher' wavenumber band found in the Raman spectra is inactive for IR spectroscopy, the reason why the IR band exhibits the unique higher wavenumber shift without a band splitting has readily been revealed. 
Associated Content: The Supporting Information is available free of charge on the ACS Publications

website at DOI: $\operatorname{xxxxxx}$

Calculated Raman Spectra of MA-Rfn $(n=3,5,7$ and 9), Vibrational images of the split two bands for $n=7$

Acknowledgment: This work was financially supported by Grant-in-Aid for Scientific Research (B) (No. 23350031) and Grant-in-Aid for Young Scientists (B) (No. 17K14502) from Japan Society for the Promotion of Science, and Priority Areas (23106710) from the Ministry of Education, Science, Sports, Culture, and Technology, Japan. This work was supported by the Collaborative Research Program of Institute for Chemical Research, Kyoto University (grant \#2012-38).

\section{References and Notes:}

1. Hasegawa, T. Understanding of the intrinsic difference between normal- and perfluoro-alkyl compounds toward total understanding of material properties. Chem. Phys. Lett., 2015, 627, 64-66.

2. Hasegawa, T. Physicochemical Nature of Perfluoroalkyl Compounds Induced by Fluorine. Chem. Rec. 2017, 17, in press. (DOI: 10.1002/tcr.201700018)

3. Hasegawa, T.; Shimoaka, T.; Shioya, N; Morita, K.; Sonoyama, M.; Takagi, T.; Kanamori, T. Stratified Dipole-Arrays Model Accounting for Bulk Properties Specific to Perfluoroalkyl Compounds. ChemPlusChem, 2014, 79, 1421-1425.

4. Hasegawa T., Quantitative Infrared Spectroscopy for Understanding of a Condensed Matter; Springer; Tokyo, Japan, 2017. 
5. Schachtschneider, J. H.; Snyder, R. G. Vibrational analysis of the n-paraffins-II. Normal co-ordinate calculations. Spectrochim. Acta 1963, 19, 117-168.

6. Higgs, P. W. The Vibration Spectra of Helical Molecules: Infra-Red and Raman Selection Rules, Intensities and Approximate Frequencies. Proc. Roy. Soc. A, 1953, 220, 472-485.

7. Krimm, S.; Liang, C.Y.; Surtherland, G. B. B. M. Infrared Spectra of High Polymers. II. Polyethylene. J. Chem. Phys., 1956, 23(3), 549-562.

8. Snyder, R. G. Vibrational Spectra of Crystalline n-Paraffins Part I. Methylene Rocking and Wagging Modes. J. Mol. Spectroscopy. 1960, 4, 411-434.

9. Nielsen, J. R.; Woollett, A. H. Vibrational Spectra of Polyethylenes and Related Substances. J. Chem. Phys. 1957, 26, 1391-1400.

10. Tasumi, M.; Shimanouchi, T.; Miyazawa, T. Normal Vibrations and Force Constants of Polymethylene Chain. J. Mol. Spectrosc. 1962, 9, 261-287.

11. Tasumi, M.; Krimm, S. Crystal Vibrations of Polyethylene. J. Chem. Phys. 1967, 46, 755-766

12. Brown, R. G. Raman Spectra of Polyethylenes. J. Chem. Phys. 1963, 38, 221-225.

13. Snyder, R. G. Interpretation of the Raman Spectrum of Polyethylene and Deuteropoiyethylene. $J$. Mol. Spectrosc. 1970, 36, 222-231.

14. Wilson Jr., E. B.; Decius, J. C.; Cross, P. C., Molecular Vibrations: The Theory of Infrared and Raman Vibrational Spectra; Dover; Toronto, Canada, 1955.

15. Nakamoto, K., Infrared and Raman Spectra of Inorganic and Coordination Compounds; Wiley; Hoboken, NJ, 2009. 
16. Krafft, M. P.; Riess, J. G. Chemistry, Physical Chemistry, and Uses of Molecular Fluorocarbon-Hydrocarbon Diblocks, Triblocks, and Related CompoundssUnique "Apolar" Components for Self-Assembled Colloid and Interface Engineering. Chem. Rev. 2009, 109, $1714-1792$.

17. Hirakawa, S., Takemura, T. Transitions and Phases of Polytetrafluoroethylene. Jpn. J. Appl. Phys. 1969, 8, 635-641.

18. Rabolt, J. F.; Piermarini, G.; Block, S. Raman spectroscopic evidence for conformational deformation in the high pressure phase of polytetrafluoroethylene. J. Chem. Phys. 1978, 69(6), $2872-2876$.

19. Clark, E. S. The molecular conformations of polytetrafluoroethylene: forms II and IV. Polymer 1999, 40, 4659-4665;

20. Pádua, A. A. Torsion Energy Profiles and Force Fields Derived from Ab Initio Calculations for Simulations of Hydrocarbon-Fluorocarbon Diblocks and Perfluoroalkylbromides. J. Phys. Chem. A 2002, 106, 10116-10123.

21. Koenig, J. L.; Boerio, F. J. Raman Scattering and Band Assignments in Polytetraftuoroethylene. J. Chem. Phys. 1969, 50(7), 2823-2829.

22. Hannon, M. J.; Boerio, F. J.; Koenig, J. L. Vibrational Analysis of Polytetraftuoroethylene. J. Chem. Phys. 1969, 50(7), 2829-2836.

23. Piseri, L.; Powell, B. M.; Dolling, G. Lattice dynamics of polytetrafluoroethylene. J. Chem. Phys. 1973, 58(1), 158-171.

24. Rabolt, J. F.; Fanconi, B. Raman Scattering from Finite Polytetrafluoroethylene Chains and a Highly Oriented TFE-HFP Copolymer Monofilament. Macromolecules, 1978, 11(4), 740-745. 
25. Hasegawa, T.; Shimoaka, T.; Tanaka, Y.; Shioya, N; Morita, K.; Sonoyama, M.; Amii, H.; Takagi, T.; Kanamori, T. An Origin of Complicated Infrared Spectra of Perfluoroalkyl Compounds Involving a Normal Alkyl Group. Chem. Lett. 2015, 44, 834-836.

26. Gaussian 09, Revision D.01, Frisch, M. J.; Trucks, G. W.; Schlegel, H. B.; Scuseria, G. E.; Robb, M. A.; Cheeseman, J. R.; Scalmani, G.; Barone, V.; Mennucci, B.; Petersson, G. A.; , et al. Gaussian, Inc., Wallingford CT, 2009.

27. Becke, A. D. Density-functional thermochemistry. III. The role of exact exchange. J. Chem. Phys. 1993, 98, 5648-5652.

28. Lee, C.; Yang, W.; Parr, G. R.; Development of the Colle-Salvetti correlation-energy formula into a functional of the electron density. Phys. Rev. B 1988, 37, 785-789.

29. Foresman, J. B.; Frisch, A. Exploring Chemistry with Electronic Structure Methods: A Guide to Using Gaussian; Gaussian, Pittsburg, PA, 1995.

30. Shimoaka, T.; Tanaka, Y.; Shioya, N; Morita, K.; Sonoyama, M.; Amii, H.; Takagi, T.; Kanamori, T.; Hasegawa, T. Surface properties of a single perfluoroalkyl group on water surfaces studied by surface potential measurements. J. Colloid Interf. Sci. 2016, 483, 353-359. 


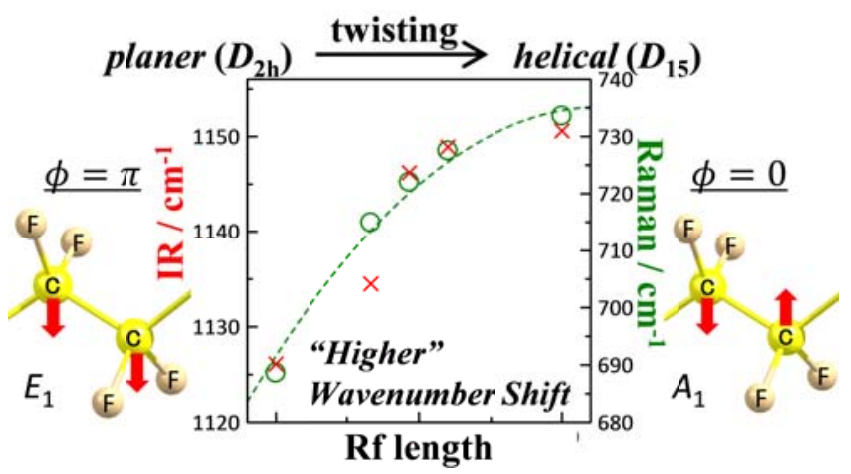

TOC Graphic 